\title{
PkwA, a WD-repeat protein, is expressed in spore-derived mycelium of Thermomonospora curvata and phosphorylation of its WD domain could act as a molecular switch
}

\author{
Bharat Joshi, Lubomir Janda, Zoia Stoytcheva and Pavel Tichy \\ Author for correspondence: Pavel Tichy. Tel: +420 2475 2356. Fax: +420 24752347. \\ e-mail:tichy@biomed.cas.cz
}

Laboratory of Physiology and Genetics of

Actinomycetes, Institute of Microbiology, AS CR,

Videnska 1083, 142 20,

Prague-4, Czech Republic

\begin{abstract}
WD-repeat proteins are found in all eukaryotes and are implicated in a variety of regulatory functions as a result of protein-protein interactions. PkwA from Thermomonospora curvata CCM3352 is a first potential example of a WDrepeat protein in a prokaryotic actinomycete. A mAb (3G2) was generated against the carboxy terminus of PkwA and was used to analyse the expression of PkwA in T. curvata. PkwA was detected in exponential growth phase following inoculation with spores, but could not be found at any stage of growth following inoculation with vegetative mycelium. PkwA and its WD domain were expressed in Escherichia coli as His-tag derivatives and purified on a Talon metal affinity matrix. The WD domain was phosphorylated by Pkg2, a membrane-spanning protein Ser/Thr kinase from ' Streptomyces granaticolor'. A membrane fraction from an exponential, spore-derived culture of $T$. curvata was found to phosphorylate the WD domain specifically in the presence of $\mathrm{Mn}^{2+}$. These data confirm that PkwA is expressed in spore-derived exponential growth phase of $T$. curvata and could play a role as a molecular switch in a signalling pathway.
\end{abstract}

Keywords: PkwA, WD-repeat protein, protein Ser/Thr kinase, phosphorylation, Thermomonospora curvata

\section{INTRODUCTION}

The so-called WD-repeat proteins contain four to eight copies of a highly conserved motif, which is usually flanked by the characteristic dipeptides GH (Gly-His) and WD (Trp-Asp) (Neer et al., 1994; Neer \& Smith, 1996; Smith et al., 1999). They form an ancient regulatory protein family, which was originally thought to be confined to eukaryotes (for a review, see Neer et al., 1994). The first reported WD-repeat protein was the $\beta$ subunit of a heterotrimeric GTP-binding protein (G protein) which transduces signals across the plasma membrane (Fong et al., 1986) and folds in a propellerlike structure (Neer \& Smith, 1996; Smith et al., 1999). All WD-repeat proteins in eukaryotes seem to play a regulatory role and are involved in diverse functions mediated through protein-protein interactions (Neer et al., 1994). Some interact with kinases to activate or inactivate and target them to their physiological sub-

Abbreviations: DIG, digoxigenin; MBP, maltose-binding protein; WD, Trp-Asp; WDA, WD domain of PkwA. strates (Ron et al., 1994, 1995; Kolman \& Egelhoff, 1997; Kwak et al., 1997; Datta et al., 1998; Bhalerao et al., 1999). There have been only a few reports where a single polypeptide carrying a WD-repeat domain also possesses a functional domain for an enzyme activity. These cases include MHCK (myosin heavy chain kinase) A and MHCK B reported in Dictyostelium (Futey et al., 1995; Claney et al., 1997), SPA1 (suppressor of phyA105) in Arabidopsis (Hoecker et al., 1999). MHCK A, MHCK B and SPA1 have a protein kinase domain in the amino-terminal region whilst the WD-repeat domain is located in the carboxy-terminal region.

The pkwA gene of Thermomonospora curvata CCM3352 (Janda et al., 1996) was the first reported example of a gene encoding a WD-repeat protein in prokaryotes. The DNA sequence indicated that it encodes a protein with seven tandem repeats of 31 amino acids, having the characteristic dipeptides $\mathrm{GH}$ (Gly-His) and WD (Trp-Asp). There is also a putative Ser/Thr-type kinase domain at its amino terminus, which represents the first case of a eukaryotic-type 
Ser/Thr kinase (for a review, see Zhang, 1996) with a WD repeat in actinomycetes. Interestingly, Ser/Thrtype kinases found in Streptomyces spp., for instance AfsK from Streptomyces coelicolor A3(2) (Matsumoto et al., 1994), and Pkg3, Pkg4 (Vomastek et al., 1998) and Pkg2 (Nadvornik et al., 1999) from 'Streptomyces granaticolor' show a conserved region of seven tandem repeats of 11-12 amino acids with similarity to the tryptophan-docking motif at their carboxy terminus, which could also fold into a propeller-like structure (Vomastek et al., 1998; Nadvornik et al., 1999).

In the last three years, several genes encoding WDrepeat proteins have been discovered in the genus Streptomyces, such as $w d p A$ from $S$. coelicolor A3(2) (AJ131817) and wdlA from Streptomyces lincolnensis RIA 1246 (GenBank accession no. AF116463) (both cloned, sequenced and expressed in our laboratory; unpublished data). In addition, two genes (AL078635, AL021411) have been detected during the S. coelicolor A3(2) sequencing project. A recently found gene (AL136500) encodes a hypothetical protein very similar to PkwA. Putative WD-repeat-encoding genes in some other prokaryotes such as Synechocystis PCC6803 (Q55563), Thermotoga maritina (AE001822), Myxococcus xanthus (AF162663), Deinococcus radiodurans (AE000513) and Chlorobium tepidum have also been reported. However, all of these data are just nucleotide sequences; there is no direct evidence for the expression of WD-repeat proteins in any prokaryotes and hence their role in these organisms remains unknown. Nevertheless, the fact that a number of WD-repeat-proteinencoding genes is present in prokaryotes suggests that this ancient regulatory protein family can no longer be regarded as confined to eukaryotes. Since it is known that these proteins are involved in diverse functions, their very presence in prokaryotes may indicate novel regulatory systems.

In this paper we demonstrate the expression of $\mathrm{PkwA}$, a WD-repeat protein, in the thermophilic actinomycete $T$. curvata CCM3352. We show that the expresssion of PkwA is limited to exponential phase of spore-derived growth and that the WD domain of PkwA can undergo phosphorylation in the presence of membrane fractions of T. curvata.

\section{METHODS}

Overexpression and purification of the carboxy-terminal part of PkwA. DNA manipulations were carried out according to the standard procedures described by Sambrook et al. (1989). Escherichia coli NM522 (Promega) was used as a recipient strain in all cases. Plasmid pMPT2 [pTZ19R (USB) carrying $3.2 \mathrm{~kb}$ SalI fragment of $T$. curvata chromosomal DNA] (Janda et al., 1996) (Fig. 1) with the $p k w A$ and $p k w R$ genes was shortened using the restriction enzyme SmaI. The resulting plasmid was named pMPT2-S (Fig. 1). This carries only a fragment of the $p k w A$ gene encoding the WD repeats plus 65 amino acid residues from the spacer region. This fragment was recloned in the pMAL-c2 expression system (New England Biolabs) using restriction enzymes EcoRI and HindIII. The fusion between the gene encoding maltosebinding protein (MBP) and the WD-repeat-encoding part of

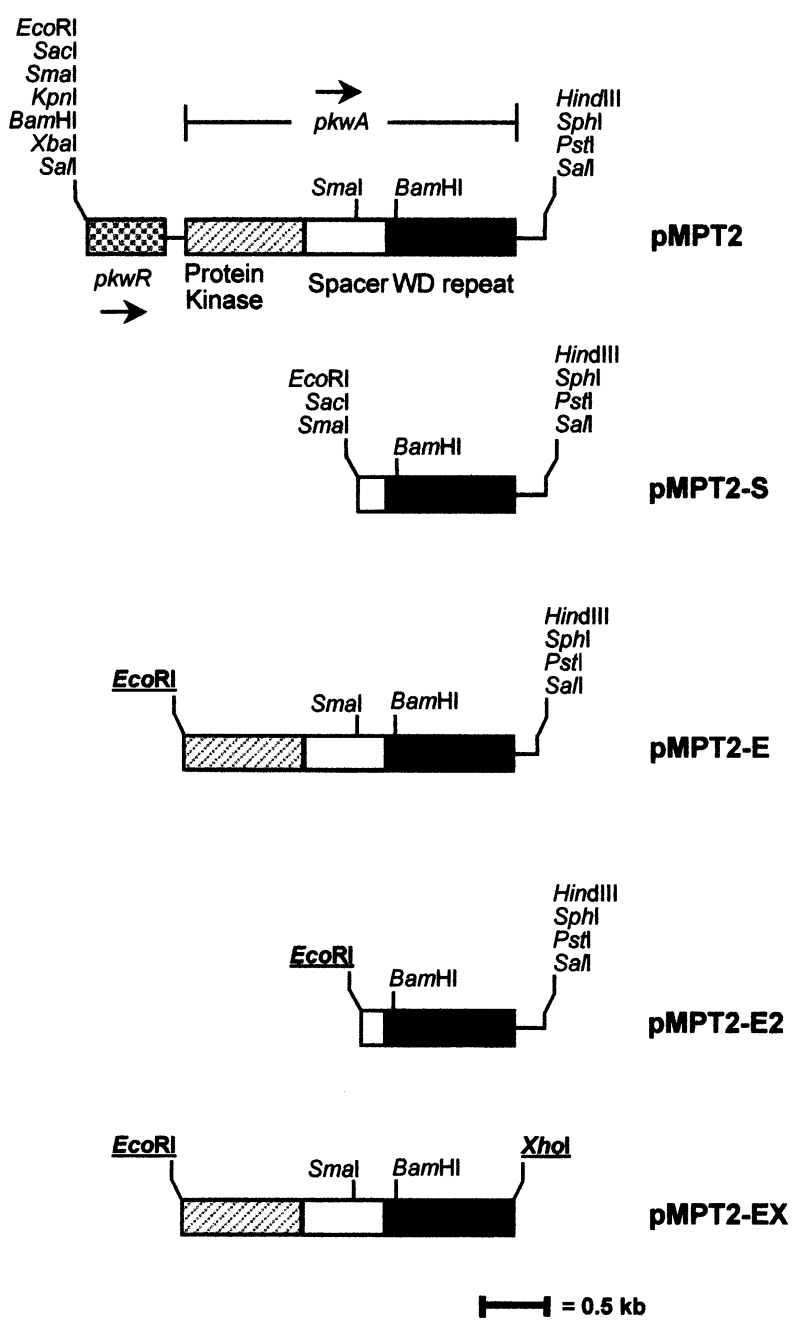

Fig. 1. Plasmid constructs used in overexpression experiments. Newly introduced restriction sites are shown in bold and underlined.

$p k w A$ was confirmed by sequencing. Overexpression and purification of the fusion protein on an amylose affinity matrix were performed according to a standard protocol (New England Biolabs). The fusion protein was digested with Factor $\mathrm{Xa}$ (New England Biolabs) and the two proteins [MBP $(42 \mathrm{kDa})$ and the carboxy-terminal part of PkwA (45.5 kDa)] were separated on an amylose affinity matrix (New England Biolabs) and Sephacryl S-200 HR (Amersham Pharmacia Biotech) into two fractions according to the suppliers' protocols. The fraction with the carboxy-terminal part of PkwA was used for immunization.

mAb preparation. Two female BALB/c mice were immunized intraperitonealy at $14 \mathrm{~d}$ intervals with three doses ( $40 \mu \mathrm{g}$ each) of the carboxy-terminal part of the PkwA protein $(45.5 \mathrm{kDa})$ electroblotted on a nitrocellulose disc. The disc was homogenized and injected into the mice. Fourteen days after the third dose and four days before fusion, mice were given an intravenous booster dose of $120 \mu \mathrm{g}$ protein. Fusion with a Sp2/mIL-6 plasmocytoma cell line (Harris et al., 1992), hybridoma cloning and production of ascetic fluid were performed according to the standard protocol (Galfre et al., 1977). mAbs were screened using ELISA and Western blotting. 
Preparing constructs for PkwA and WDA expression in E. coli. A plasmid that expresses full-length PkwA (residues 1-742) in E. coli with a hexa-His-tag at the carboxy terminus was constructed. An EcoRI site at the start of $p k w A$ was generated in pMPT2 by PCR and the resulting construct was named pMPT2-E (Fig. 1). The suitable restriction sites XhoI and HindIII were also generated and the termination codon was removed from the $3^{\prime}$ end of $p k w A$ in pMPT2-E by PCR. The $2.2 \mathrm{~kb}$ EcoRI-XhoI fragment carrying $p k w A$ was then subcloned in pET24a $(+)$ (Novagen) and named pMPT2-EX (Fig. $1)$. In this construct there are an extra 14 codons from the vector at the $\mathrm{N}$ terminus of $\mathrm{PkwA}$.

A plasmid that expresses a His-tag derivative of the WD domain of PkwA (WDA) was also constructed. The EcoRI site was generated at the 3300 position in pkwA (GenBank accession no. AF115313) by PCR using pMPT2-E (Fig. 1) as template. The amplified fragment $(\sim 1.0 \mathrm{~kb})$ was digested with EcoRI/HindIII, subcloned in pET28b $(+)$ (Novagen) and named pMPT2-E2 (Fig. 1). This construct contains an extra 36 codons from the vector at the $\mathrm{N}$ terminus of the WD domain, which includes a hexa-His-tag and a T7-tag.

Expression and purification of PkwA and WDA. Both pMPT2EX and pMPT2-E2 constructs were individually transformed into E. coli strain BL21 (DE3) (Novagen). Transformants were grown at $37^{\circ} \mathrm{C}$ in $\mathrm{LB}$ medium containing $50 \mu \mathrm{g}$ kanamycin $\mathrm{ml}^{-1}$ until the optical density at $600 \mathrm{~nm}$ reached 0.6 . Cells were induced with $1 \mathrm{mM}$ (final concentration) IPTG for $3 \mathrm{~h}$ and then placed on ice for $5 \mathrm{~min}$; they were then harvested by centrifugation at $13000 \mathrm{~g}$ (BiofugeA; Heraeus Sepatech). Cells were disrupted by sonication and native protein purification of both PkwA and WDA His-tag derivatives was performed on a metal affinity matrix according to the standard protocols supplied with the Talon metal affinity resin (Clontech).

Maintenance and growth curve of T. curvata CCM3352. T. curvata strain CCM3352 was obtained from the Czechoslovak Culture Collection (Brno, Czech Republic) and maintained on SPTC agar medium as described by Janda et al. (1997). Cultivation and growth measurements of T.curvata in mineral salt-vitamin minimal medium (MM-medium) was performed as described by Petricek et al. (1989).

Detection of PkwA in T. curvata CCM3352. To examine PkwA expression in T. curvata, spores stored in $20 \%$ (v/v) glycerol at $-70{ }^{\circ} \mathrm{C}$ were inoculated $\left(10^{8}\right.$ spores in $100 \mathrm{ml}$ medium $)$ into MM-medium (Petricek et al., 1989) and incubated on an orbital shaker (Gallenkamp) (200 r.p.m.) at $52^{\circ} \mathrm{C}$ in a $500 \mathrm{ml}$ Erlenmeyer flask. Samples were collected at $6 \mathrm{~h}$ intervals. Pellicles were harvested by centrifugation $(35000 \mathrm{~g}, 15 \mathrm{~min}$ ) (SS34, RT5C Sorvall; Dupont), washed once with ice-cold Xbuffer $(20 \mathrm{mM}$ Tris $/ \mathrm{HCl}$ pH 7.5 containing $1 \mathrm{mM}$ DTT, $1 \mathrm{mM}$ EDTA and $0.1 \mathrm{mM}$ PMSF) and stored frozen at $-20^{\circ} \mathrm{C}$. The samples were resuspended in ice-cold X-buffer ( $\sim 50 \mathrm{mg}$ in $0.5 \mathrm{ml}$ ) and disrupted by ultrasonic treatment (Labsonic 2000; USA). Each cycle consisted of sonication for $1 \mathrm{~min}$ followed by cooling for $2 \mathrm{~min}$. These two steps were repeated until the pellicles were completely disrupted. The whole sonication process was carried out on ice. The lysate was centrifuged and supernatant was separated. The crude extract was boiled for 3 min with SDS-PAGE loading buffer and the proteins were separated on $0.1 \%$ SDS $/ 10 \%$ acrylamide PAGE gels (Laemmli, 1970). The gel was electroblotted onto Hybond $\mathrm{C}$ nitrocellulose membrane (Amersham) using Tris/glycine/methanol buffer ( $\mathrm{pH} 8.0)$ and $150 \mathrm{~mA}$ constant current for $2.5 \mathrm{~h}$. PkwA was probed using anti-PkwA mAb $3 G 2(1: 100)$ and the signal was developed by horse-radishperoxidase-labelled anti-mouse antibody $(1: 1000)$ (Sevac) as described in the standard ECL (enhanced chemiluminescence) protocol manual (Amersham).

Total RNA extraction from $\boldsymbol{T}$. curvata. Total RNA from 18, 24, 30, 36 and $48 \mathrm{~h}$ spore-derived cultures of T. curvata was prepared as described by Petricek et al. (1992). The integrity of RNA was checked on agarose electrophoresis containing 2.2 $\mathrm{M}$ formaldehyde.

Preparation of digoxigenin (DIG)-labelled RNA probe by in vitro transcription. The $1.4 \mathrm{~kb}$ EcoRI-BamHI fragment (pMPT2-E, Fig. 1) from $p k w A$ was subcloned in pGEM-4Z (Promega) and the resulting plasmid was named pGEMEB. pGEMEB was linearized by SmaI, separated by electrophoresis, isolated from the agarose gel and used for in vitro transcription. The reaction was performed according to the standard procedure supplied with the DIG RNA Labelling Kit (SP6/T7) (Roche Molecular Biochemicals) using $1 \mu \mathrm{g}$

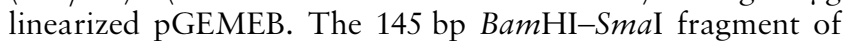
$p k w A$ was transcribed in the opposite direction using the T7 promoter and T7 RNA polymerase.

Northern hybridization. Samples of total RNA $(\sim 25 \mu \mathrm{g})$ were denatured, separated by $2.2 \mathrm{M}$ formaldehyde-agarose gel electrophoresis and vacuum-blotted onto Amersham Hybond $\mathrm{N}+$ membranes following the procedure of Bormann et al. (1992). RNA-RNA hybridization and disodium 3-(4-methoxyspiro $\left\{1,2\right.$-dioxetane-3, $2^{\prime}$ - $\left(5^{\prime}\right.$-chloro $)$ tricyclo[3.3.1.1 ${ }^{3,7}$ ]decan $\left.\}-4-y l\right)$ phenyl phosphate (CSPD)-based chemiluminescent detection were performed with the DIG Luminescent Detection Kit for Nucleic Acids (Boehringer Mannheim), following the standard procedure recommended by the suppliers.

CDNA synthesis and PCR amplification. cDNA was synthesized in $20 \mu \mathrm{l}$ of a reaction mixture using a sequence-specific primer WD-Rev2 (5' AGG GTT GTG TGT TCT TC 3') annealing at nucleotides 4150-4166 of pkwA (GenBank accession no. AF115313) and MMLV reverse transcriptase (Amersham Pharmacia Biotech) according to the standard procedure described in the PCR Application Manual (Boehringer Mannheim). A $5 \mu \mathrm{l}$ aliquot from the first-strand cDNA synthesis mixture was subjected to 30 cycles of amplification by PCR. In addition to amplification buffer and template cDNA, the amplification mixture contained (in a final volume of $100 \mu \mathrm{l}$ ) $2.5 \mathrm{U} \mathrm{Taq}$ polymerase (Promega), $1.5 \mathrm{mM} \mathrm{MgCl}_{2}$, $250 \mu \mathrm{M}$ of each deoxynucleotide triphosphate and $50 \mathrm{pmol}$ forward primer WD-For1 (5' AAC ACG CCG TCC TCA AA $3^{\prime}$ ) annealing at nucleotides 3779-3795 of $p k w A$. Reverse primer WD-Rev2 was added to a final concentration in the reaction mixture of $50 \mathrm{pmol}$.

Assessing the effect of the WD domain on Pkg2. E. coli strain BL21 (DE3) harbouring pEX2, which encodes Pkg2, was grown and induced by a method similar to that described for PkwA and WDA. The induced E. coli cells from $1 \mathrm{ml}$ culture were harvested, washed three times in ice-cold phosphorylation buffer $(50 \mathrm{mM}$ Tris $/ \mathrm{HCl} \mathrm{pH} 7.5$ containing $50 \mathrm{mM}$ $\mathrm{NaCl}, 10 \mathrm{mM} \mathrm{MgCl}_{2}, 1 \mathrm{mM}$ EDTA and $10 \mathrm{mM} \beta$ mercaptoethanol), resuspended in $150 \mu \mathrm{l}$ of the same buffer and disrupted by sonication. The lysate was centrifuged $(18000 \mathrm{~g})$ at $4{ }^{\circ} \mathrm{C}$ for $10 \mathrm{~min}$. The supernatant was collected and used as the source of Pkg2. Partially purified His-tag derivatives of both PkwA and WDA were transferred in icecold phosphorylation buffer using microcon-30 (Amicon). $\mathrm{PkwA}$, WDA and Pkg2 were subjected to autophosphorylation as controls. The reaction was carried out at $37^{\circ} \mathrm{C}$ for $10 \mathrm{~min}$ after mixing $5 \mu \mathrm{Ci}\left(1.85 \times 10^{5} \mathrm{~Bq}\right)\left[\gamma^{32} \mathrm{P}\right] \mathrm{ATP}$ (Amersham Pharmacia Biotech). To assess the effect of the WD domain, PkwA $(\sim 1.0 \mu \mathrm{g})$ and WDA $(\sim 3.0$ and $\sim 5.0 \mu \mathrm{g})$ were separately mixed with $\mathrm{Pkg} 2$ and subjected to phosphorylation. 
All the above reactions were terminated by adding SDS-PAGE loading buffer. After electrophoresis, gels were soaked in boiling 16\% trichloroacetic acid (Mannai \& Cozzone, 1982), dried under vacuum and exposed to an image processor (Fuji film). The signal was developed with Fuji-BAS-5000 (Fuji film).

WDA phosphorylation in T. curvata. Twenty-four-hour sporederived pellicles of T. curvata $(\sim 50 \mathrm{mg})$ were harvested and washed three times in ice-cold phosphorylation buffer $(500 \mu \mathrm{l}$ each time), resuspended in $500 \mu \mathrm{l}$ of the same buffer and disrupted by sonication as described above. The lysate was ultracentrifuged $(100000 \mathrm{~g})$ at $4{ }^{\circ} \mathrm{C}$ for $60 \mathrm{~min}$. The supernatant (cytoplasmic fraction) was collected separately. The sediments containing membrane fractions were washed three times in ice-cold phosphorylation buffer $(500 \mu \mathrm{l}$ each time $)$ and resuspended in $100 \mu \mathrm{l}$ of the same buffer. Both cytoplasmic fraction and membrane fractions were subjected to autophosphorylation as negative controls. To assess WDA phosphorylation, $\sim 2.0 \mu \mathrm{g}$ purified WDA was mixed separately with $5 \mu \mathrm{l}$ cytoplasmic fraction and $5 \mu \mathrm{l}$ membrane fractions of $T$. curvata, and phosphorylation was carried out by mixing with $5 \mu \mathrm{Ci}\left(1.85 \times 10^{5} \mathrm{~Bq}\right)\left[\gamma^{3}{ }^{32} \mathrm{P}\right] \mathrm{ATP}$ (Amersham Pharmacia Biotech). Various conditions were used for both control and test samples, such as different cations, different temperatures and different times of incubation. Termination of reaction, electrophoresis, gel treatment, drying and signal development were performed as described above.

\section{RESULTS}

\section{Preparation of $\mathrm{mAbs}$}

To follow the expression of PkwA in T. curvata, we generated a specific $\mathrm{mAb}$ against the WD domain of $\mathrm{PkwA}$. It was desirable for the $\mathrm{mAb}$ to react specifically with the WD-repeat domain of the protein rather than with the kinase domain. Hence, the $3^{\prime}$ end of the gene carrying the WD-repeat region and part of the spacer region (Fig. 1) was subcloned into the expression vector pMAL-c2 to produce a fusion protein with MBP. This protein was overexpressed in E. coli NM522 and purified on an amylose affinity matrix (Fig. 2a, lanes 1, 2, 3). After digesting purified MBP-WD with Factor Xa, the WD-repeat part of the fusion protein could be separated from MBP (Fig. 2a, lane 4) and purified (Fig. 2a, lane 5). This protein was used to generate mAbs. A number of mAbs from several clones strongly reacted with the fused MBP-WD-repeat protein (Fig. 2b, lanes 1). But, with the exception of 3G2 and 2H7 (Fig. 2b, lanes 3), they also cross-reacted with purified MBP alone (Fig. 2b, lanes 2). Since only $\mathrm{mAb} 3 \mathrm{G} 2$ reacted with the purified His-tag derivative of PkwA (Fig. 2c, lane 1), it was used for further experiments.

\section{PkwA expressed as a His-tag derivative}

The purpose of expressing $\mathrm{PkwA}$ in E. coli was to produce larger amounts of protein for its characterization, and to use it as a substrate for phosphorylation. Its His-tag derivative was intended to facilitate purification. Hence, E. coli strain BL21 (DE3) harbouring pMPT2-EX (Fig. 1) was subjected to induction under the control of the T7 promoter. No IPTG-responsive PkwA band could be seen in the SDS- (a)

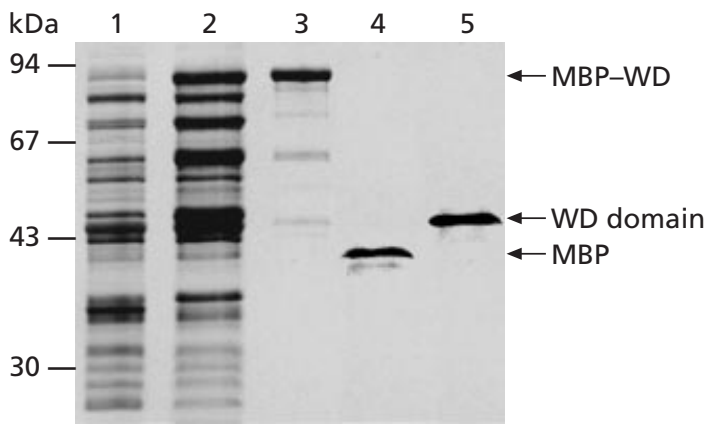

(b)

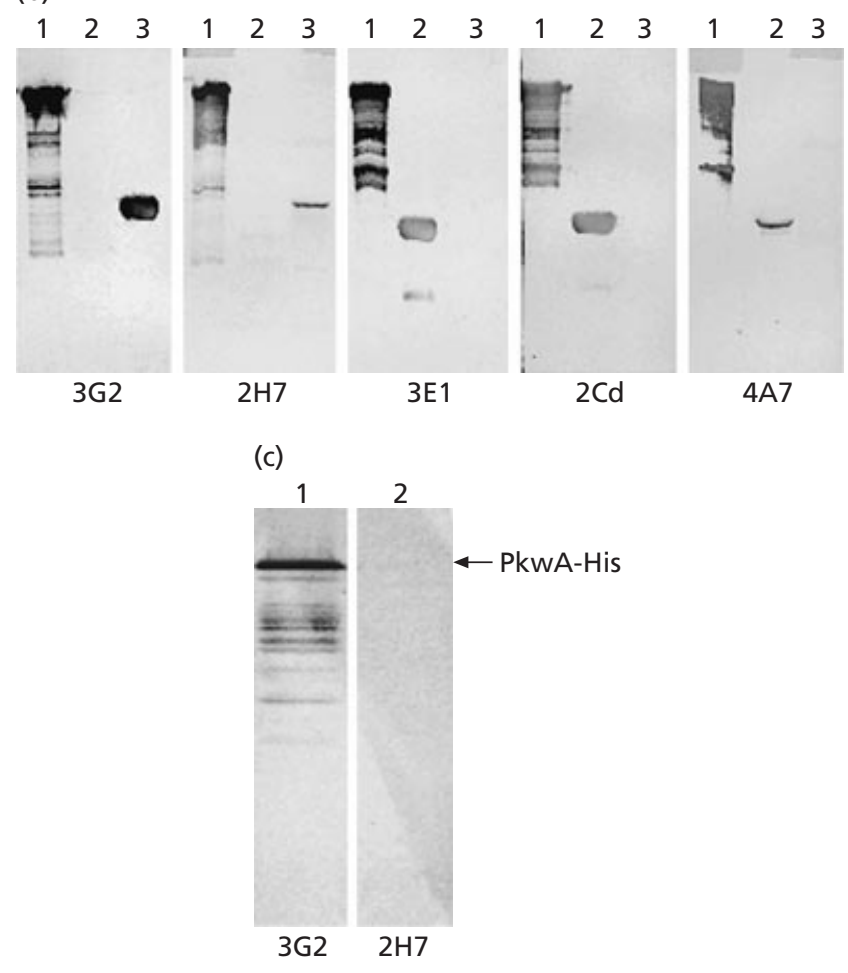

Fig. 2. (a) Coomassie-blue-stained gel showing expression and purification of the WD domain of PkwA. Lanes 1 and 2, uninduced (lane 1) and IPTG-induced (lane 2) E. coli NM522 crude cell extracts harbouring PMAL-C2 with the WD-encoding fragment. Overexpressed $\sim 90 \mathrm{kDa}$ MBP-WD fusion protein is visible in lane 2. Lane 3, amylose affinity-purified MBP-WD. After digestion with Factor $\mathrm{Xa}, \mathrm{MBP}$ and WD domain were separated by amylose affinity and gel filtration. Lane 4, purified MBP at $\sim 42 \mathrm{kDa}$; lane 5, purified WD domain at $\sim 45.5 \mathrm{kDa}$. (b) Western blots showing the specificity of mAbs. Lanes: 1, MBP-WD fusion protein; 2, purified MBP; 3, purified WD domain. 3G2, 2H7, 3E1, $2 \mathrm{Cd}$ and $4 \mathrm{~A} 7$ represent several $\mathrm{mAb}$ clones, each reacting with MBP-WD. Only $3 \mathrm{G} 2$ and $2 \mathrm{H} 7$ react with purified WD domain, whereas 3E1, 2Cd and 4A7 crossreact with MBP. (c) Western blot showing attempts to detect recombinant PkwA expressed in E. coli with $3 G 2$ (lane 1) and $2 \mathrm{H} 7$ (lane 2). A protein of $\sim 100 \mathrm{kDa}$ and some low-molecularmass bands were detected by $3 \mathrm{G} 2$, but not by $2 \mathrm{H} 7$.

PAGE gel stained with Coomassie blue (Fig. 3a, lane 2). However, when the Western blot of the gel was probed with the mAb 3G2, a strong positive signal at an apparent molecular mass of $\sim 100 \mathrm{kDa}$ was detected in 
(a)

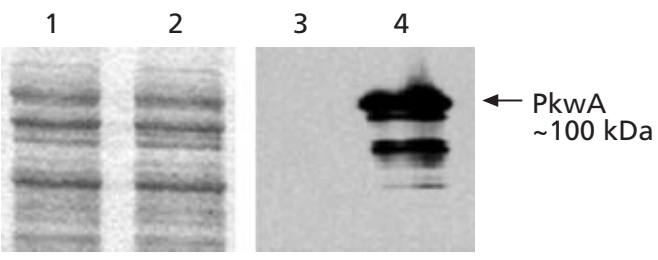

(b)

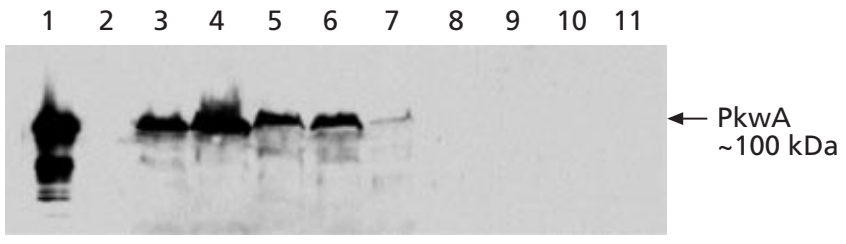

Fig. 3. Overexpression of PkwA His-tag derivative. (a) PkwA overexpression after induction is not visible on SDS-PAGE gels. Lanes 1 and 2, uninduced (lane 1) and IPTG-induced (lane 2) E. coli BL-21 (DE3) crude cell extract harbouring PMPT2-EX (no IPTG-responsive band in SDS-PAGE gel). Corresponding Western blot (lane 3, uninduced; lane 4, induced) and mAb $3 G 2$ detection show expression of PkwA at $\sim 100 \mathrm{kDa}$. (b) Western blot showing $\mathrm{mAb} 3 \mathrm{G} 2$ detection of PkwA in T. curvata and its overexpression in $E$. coli BL-21 (DE3). Lanes: 1 and 2, IPTGinduced (lane 1) and uninduced (lane 2) E. coli BL-21 (DE3) crude cell extract harbouring PMPT2-EX (overexpressed PkwA at $\sim 100 \mathrm{kDa}) ; 3-7, T$. curvata crude extract from spore-derived cultures after 18,24, 30, 36 and $48 \mathrm{~h}$ of growth, respectively; 8 , empty; 9-11, T. curvata crude extract after 18, 24 and $30 \mathrm{~h}$ of growth, respectively, from cultures inoculated with vegetative mycelium. The same amount of total protein was applied in each lane. $100 \mathrm{kDa}$ PkwA is observed in 18, 24, 30 and $36 \mathrm{~h}$ samples but almost disappears after $48 \mathrm{~h}$ of spore-derived growth. No PkwA was observed at any stage of growth initiated by vegetative mycelium. T. curvata PkwA (lane 3 ) and recombinant PkwA (lane 1) have similar mobility.

the lysate from the induced culture (Fig. 3a, lane 4) but not in the uninduced culture sample (Fig. 3a, lane 3). There were also weaker bands of lower molecular mass. The observed molecular mass of recombinant PkwA is somewhat higher than that deduced $(\sim 80 \mathrm{kDa})$ from the amino acid sequence (742 residues) of the $p k w A$ gene (Janda et al., 1996).

\section{PkwA is expressed in T. curvata CCM3352}

Crude protein extracts of T. curvata samples harvested at different times of growth after spore germination were separated by SDS-PAGE and used for Western blotting. A strong band with an apparent molecular mass of about $100 \mathrm{kDa}$ (Fig. 3b, lanes 3-6) was repeatedly detected by mAb 3G2 after 18, 24, 30 and 36 $\mathrm{h}$, while there was active growth. The signal became weaker at $48 \mathrm{~h}$ (lane 7), which was about $8 \mathrm{~h}$ after the dry weight of the culture had stopped increasing. The slow migration of PkwA from T. curvata on SDS-PAGE gels was however very much in agreement with the expressed recombinant PkwA in E. coli (Fig. 3b, lanes 3 and 1). Interestingly, no PkwA expression was observed at any stage of growth $(18,24$ or $30 \mathrm{~h}$ ) when vegetative mycelium that had grown for $30 \mathrm{~h}$ was used as the inoculum (Fig. 3b, lanes 9-11). (a)

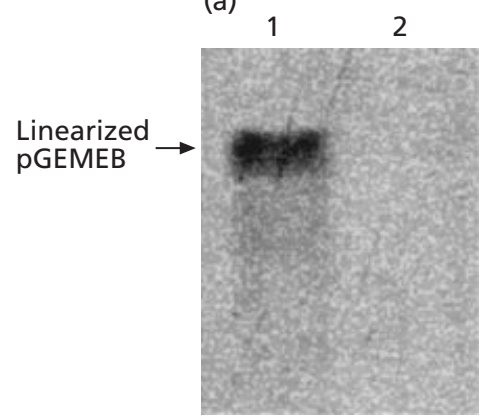

(b)

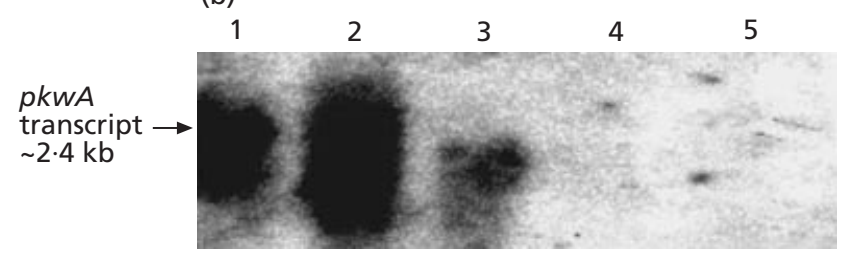

(c)

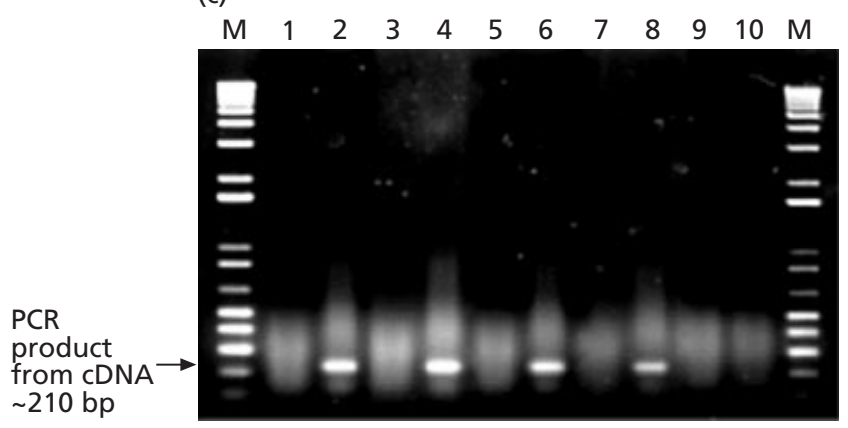

Fig. 4. Northern hybridization and RT-PCR show the presence of pkwA transcript. (a) Specificity of the DIG-labelled RNA probe. Lanes: 1 , linearized PGEMEB; 2 , linearized pGEM-4Z as a negative control. DIG-labelled RNA probe specific to $p k w A$ hybridizes with linearized pGEMEB but not with pGEM-4Z. (b) Northern hybridization. Lanes 1-5, total RNA samples extracted after $18,24,30,36$ and $48 \mathrm{~h}$ of growth, respectively. The DIGlabelled RNA probe hybridized with the pkwA transcript of $2.4 \mathrm{~kb}$ in total RNA extracted after 18,24 and $30 \mathrm{~h}$ of growth. (c) PCR amplification from cDNA of a $\sim 210$ bp product. Lanes: $\mathrm{M}$, size marker (12000-100 bp); 1, 3, 5, 7 and 9, controls without MMLV reverse transcriptase (total RNA samples extracted after $18,24,30,36$ and $48 \mathrm{~h}$ of growth, respectively, subjected to CDNA synthesis followed by PCR); $2,4,6,8$ and 10, total RNA samples extracted after 18, 24, 30, 36 and $48 \mathrm{~h}$ of growth, respectively, subjected to CDNA synthesis with MMLV reverse transcriptase. $A \sim 210$ bp PCR-amplified product is visible in all CDNAs synthesized from total RNA samples, except those extracted after $48 \mathrm{~h}$ of growth and controls.

\section{Northern analysis and RT-PCR further confirm PkwA expression}

To further analyse $p k w A$ expression, a $p k w A$-specific DIG-labelled RNA probe, generated by in vitro transcription, was used in Northern analysis. Prior to hybridization, the specificity of the probe for $p k w A$ was determined against linearized pGEMEB by DNA-RNA hybridization (Fig. 4a, lane 1). After 18 and 24 h growth, RNA from $T$. curvata showed a strong signal at approximately $2.4 \mathrm{~kb}$, decreasing after $30 \mathrm{~h}$ growth (Fig. 
(a)

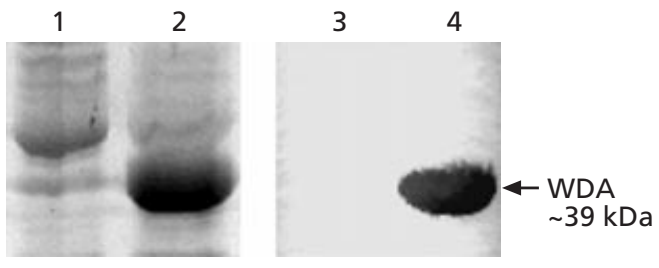

(b)

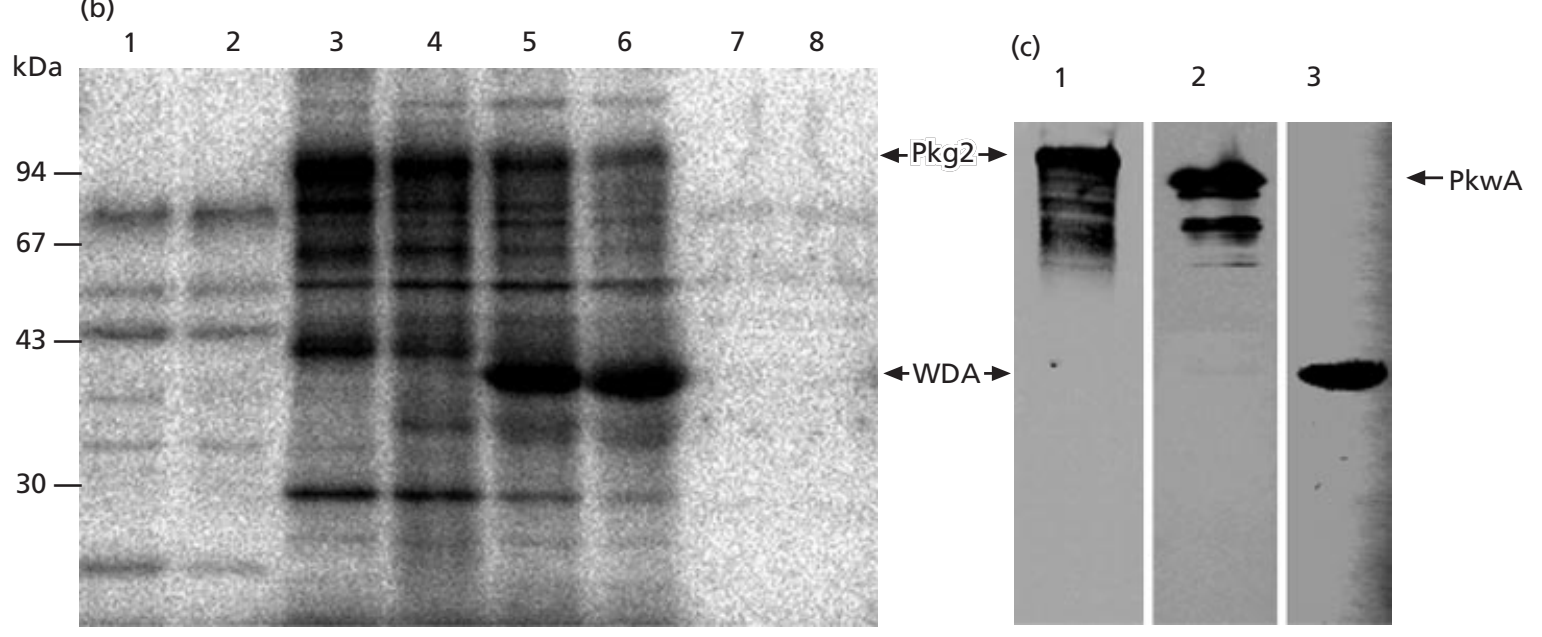

(d)

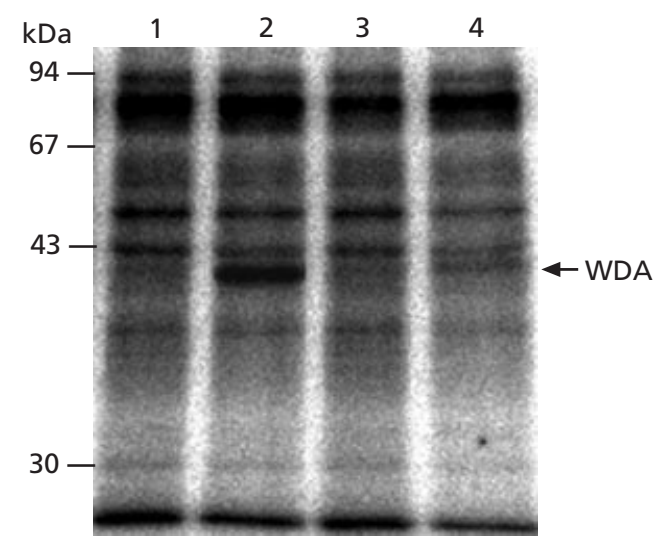

Fig. 5. Overexpression of WDA as a His-tag derivative and its phosphorylation analysis. (a) Overexpression of WDA. Lanes 1 and 2, uninduced (lane 1) and IPTG-induced (lane 2) E. coli BL-21 (DE3) crude cell extract harbouring pMPT2-E2, which encodes WDA as a His-T7-tag derivative. An IPTG-responsive band is visible at $\sim 39 \mathrm{kDa}$ in induced crude cell extract analysed on SDS-PAGE gels. Lanes 3 and 4, corresponding Western blot and mAb 3G2 detection (lane 3, uninduced; lane 4, induced) confirm overexpression. (b) Pkg2 phosphorylates WDA. Lanes 1, 2, 3, 7 and 8, controls for phosphorylation analysis. Lanes 1 and 2, induced E. coli BL21 (DE3) crude cell extracts harbouring pET28b (+) mixed with purified PkwA $(\sim 1.0 \mu \mathrm{g})$ and WDA $(\sim 3.0 \mu \mathrm{g})$, respectively. Neither were phosphorylated in $E$. coli crude extract. Lane 3, induced $E$. coli BL21 (DE3) crude cell extract harbouring pEX2 (expresses Pkg2) show active autophosporylated Pkg2. Lanes 7 and 8 , $\sim 1.0 \mu \mathrm{g}$ purified PkwA and $\sim 3.0 \mu \mathrm{g}$ purified WDA, respectively, do not show autophosphorylation. Lanes 4-6, induced E. coli BL21 (DE3) crude cell extract harbouring pEX2 with purified $\sim 1.0 \mu \mathrm{g}$ PkwA (lane 4), and with purified $\sim 3.0 \mu \mathrm{g}$ (lane 5) and $\sim 5.0 \mu \mathrm{g}$ (lane 6) WDA. The same amount of Pkg2 was used in lanes 3-6. Strongly phosphorylated WDA with molecular mass $\sim 39 \mathrm{kDa}$ is visible in lanes 5 and 6 . A significant decrease in Pkg2 autophosphorylation is visible in lanes 3-6. (c) Corresponding Western blots of lanes 3, 4 and 5 shown in (b) probed with polyclonal antibody anti-Pkg2 (lane 1) and anti-WD mAb 3G2 (lanes 2 and 3). Lanes 1, 2 and 3 verify the presence of Pkg2, PkwA and WDA, respectively. (d) Membrane fractions from $T$. curvata phosphorylate WDA. Lanes 1 and 3, negative controls for phosphorylation show membrane fractions and cytoplasmic fraction from $T$. curvata, respectively. Neither shows a phosphorylated band of the molecular mass of WDA. Lanes 2 and 4, purified WDA $(\sim 2.0 \mu \mathrm{g})$ mixed with the membrane fractions and cytoplasmic fraction from $T$. curvata, respectively. A phosphorylated band of $\sim 39 \mathrm{kDa}$ in lane 2 represents WDA. A faint band of similar molecular mass is also visible in lane 4. 
$4 \mathrm{~b}$, lanes 1-3). These data confirm that $p k w A$ was expressed 18, 24 and $30 \mathrm{~h}$ after inoculation, but that there was little or no observed expression after 36 and $48 \mathrm{~h}$ growth (Fig. $4 \mathrm{~b}$, lanes 4 and 5 ).

To further confirm this, a sequence-specific primer, WD-Rev2, was designed and cDNA for RT-PCR was prepared. As shown in Fig. 4(c), PCR amplification of the cDNA prepared from the T. curvata total RNA samples of the $18 \mathrm{~h}$ (lane 2), $24 \mathrm{~h}$ (lane 4) and $30 \mathrm{~h}$ (lane 6) gave a PCR product of the expected size of $210 \mathrm{bp}$, amplified by WD-For1 and WD-Rev2 primers. In contrast to Northern analysis, a weak band could also be seen in the $36 \mathrm{~h}$ sample (lane 8 ). No band appeared in the $48 \mathrm{~h}$ sample (lane 10).

\section{Pkg2 phosphorylates WDA}

To examine the effect of the WD domain of PkwA on protein Ser/Thr kinase, the Pkg2 from 'S. granaticolor' (Nadvornik et al., 1999) was used as a positive control. WDA, the WD domain of PkwA, was overexpressed as a His-tag derivative in E. coli BL21 (DE3), verified on Western blot probed by mAb 3G2 (Fig. 5a, lanes 2 and 4) and partially purified using a Talon metal affinity matrix. In a control phosphorylation reaction, PkwA and WDA did not undergo autophosphorylation (Fig. $5 \mathrm{~b}$, lanes 7 and 8) whereas the active Pkg2 was positively phosphorylated (Fig. 5b, lane 3). When Pkg2 was subjected to phosphorylation in the presence of partially purified PkwA or WDA, a decrease in the autophosphorylation ability of $\mathrm{Pkg} 2$ was reproducibly observed (Fig. 5b, lanes 4-6). At the same time, Pkg2 phosphorylated WDA (Fig. 5b, lanes 5 and 6). To rule out the possibility that this phosphorylation of WDA was due to an E. coli endogenous kinase, WDA was incubated under the same conditions with E. coli BL21 (DE3) crude extract. However, the WDA was not phosphorylated (lane 2), thus proving that it is $\mathrm{Pkg} 2$ that specifically phosphorylates WDA. Fig. 5(c), showing a Western blot of lanes 3, 4 and 5 from Fig. 5(b) run in parallel in the SDS-PAGE gels, demonstrates the presence of corresponding proteins in the reaction mixtures.

\section{Membrane fractions of $T$. curvata phosphorylate WDA}

Encouraged by the phosphorylation of WDA by Pkg2, we looked for a similar putative kinase in T. curvata. Partially purified WDA was mixed separately with the cytoplasmic fraction and the membrane fractions obtained from the spore-derived culture of T. curvata. Under a variety of conditions, phosphorylation assays were carried out. In the presence of $\mathrm{Mn}^{2+}(10 \mathrm{mM})$ at $37^{\circ} \mathrm{C}$ for 10 min with $5 \mu \mathrm{Ci}\left(1.85 \times 10^{5} \mathrm{~Bq}\right)\left[\gamma^{32} \mathrm{P}\right] \mathrm{ATP}$, a positive band of $\sim 39 \mathrm{kDa}$ (the molecular size of WDA) was observed in the membrane fractions of $T$. curvata incubated with WDA (Fig. 5d, lane 2). A faint band of similar size was also observed in the cytoplasmic fraction incubated with WDA (Fig. 5d, lane 4). Neither the cytoplasmic fraction (lane 3) nor the membrane fraction controls (lane 1) showed any phosphorylated band at the position of WDA.

Phosphorylation reactions were also performed in the presence of $\mathrm{Ca}^{2+}(10 \mathrm{mM})$ or $\mathrm{Mg}^{2+}(10 \mathrm{mM})$, alone or in combination; however, the results were negative (not shown).

\section{DISCUSSION}

In an earlier study, we reported the presence of the $p k w A$ gene encoding a WD-repeat protein in T. curvata (Janda et al., 1996). It was the first of its kind found in a prokaryote. Thereafter, it was important to demonstrate that PkwA is indeed expressed. For this purpose, a mAb, 3G2, was generated against the WDrepeat domain of PkwA.

During the examination of the expression of PkwA on Western blots, it was found that $3 \mathrm{G} 2$ reacted with the exponential-growth-phase extracts of T. curvata (Fig. $3 \mathrm{~b}$, lanes 3-7) derived from spores but interestingly, no PkwA could be detected at any stage of growth initiated from vegetative mycelium (Fig. 3b, lanes 9-11). This indicates possible involvement of PkwA in some crucial spore-derived developmental stage of the growth of $T$. curvata. The pattern of PkwA expression in sporederived growth was further confirmed by Northern hybridization. Northern analysis showed the presence of $p k w A$ transcript (Fig. 4b) during early exponential growth, which was in good agreement with the results obtained by Western blot analysis. It also suggested that the transcription of $p k w A$ could be over by the end of the early exponential phase. However, the results obtained by the more sensitive RT-PCR method (Fig. $4 c)$ enabled us to detect the presence of transcript during up to $36 \mathrm{~h}$ of growth (to late exponential phase).

The apparent molecular mass of PkwA in SDS-PAGE gels was approximately $100 \mathrm{kDa}$ instead of the $80 \mathrm{kDa}$ predicted from the amino acid sequence. However, when PkwA was expressed as a His-tag derivative in $E$. coli, it also yielded a band of about $100 \mathrm{kDa}$ on Western blots (Fig. 3a, b). Such unexpectedly slow migration of protein on SDS-PAGE gels has also been reported in other cases such as Pkg2 (Nadvornik et al., 1999). Expression of recombinant PkwA could not be seen on a stained gel, but immunodetection using mAb 3G2 on Western blots confirmed expression (Fig. 3a) similar to other reported cases (Matsumoto et al., 1994; Urabe \& Ogawara, 1995).

The deduced amino acid sequence of $p k w A$ (Janda et al., 1996) suggests the presence of a putative Ser/Thr-type kinase domain at the amino terminus of PkwA. However, we failed to detect autophosphorylation under various conditions. $\mathrm{Pkg} 3$ reported from 'S. granaticolor' represents another such case (Vomastek et al., 1998). According to the primary structure, PkwA and Pkg3 belong to the family of so-called RD protein kinases, which requires phosphorylation of an activation loop between domains VII and VIII for their activation (Johnson et al., 1996). It is very likely that the kinase 
domain of PkwA could be phosphorylated and activated by some other kinase that could be functioning together with PkwA in T. curvata.

The WD-repeat proteins are also known for their interaction with kinases (Ron et al., 1994, 1995; Kolman \& Egelhoff, 1997; Kwak et al., 1997; Datta et al., 1998) and some can even exert an inhibitory effect (Bhalerao et al., 1999). A sequence alignment of the WD domain of PkwA showed significant sequence similarity with other such WD-repeat proteins. We therefore wanted to examine the possible effect of WDA on active Pkg2, a protein Ser/Thr kinase from 'S. granaticolor' (Nadvornik et al., 1999). The aim of an attempt to autophosphorylate Pkg2 with partially purified PkwA or WDA was to prove that whatever the effect may be, it is due to the WD domain. The results showed that Pkg2 autophosphorylation was lower in the presence of PkwA or its WD domain, WDA (Fig. 5b, lanes 4, 5 and 6). However, at the same time Pkg2 phosphorylated WDA (Fig. 5b, lanes 5 and 6). It is very likely that the WDA could exert a dual effect, influencing Pkg2 autophosphorylation and at the same time acting as a substrate for Pkg2. This scenario would be similar to Skp2, $\mathrm{p} 21^{\mathrm{Cip} 1 / \mathrm{WAF} 1}$ or $\mathrm{p} 27^{\mathrm{Kip} 1}$ that inhibit CDK (cyclinedependent kinase) activity and at the same time act as substrates and undergo phosphorylation (Yam et al., 1999; Zhang et al., 1994; Sheaff et al., 1997). However, the WDA phosphorylation by $\mathrm{Pkg} 2$ encouraged us to look for the putative kinase in T. curvata. The cytoplasmic fraction and the membrane fractions obtained from 24-h-old mycelia of T. curvata were used as a possible source. When testing under various conditions in the presence of partially purified WDA, the membrane fractions of $T$. curvata showed a phosphorylated band of the molecular size of WDA (Fig. 5d, lane 2). However, the control reaction with the membrane fractions alone was negative (Fig. 5d, lane 1), implying that the phosphorylated band in the test was indeed WDA. This result was obtained in the presence of $\mathrm{Mn}^{2+}$; the reactions carried out in the presence of other cations such as $\mathrm{Ca}^{2+}$ or $\mathrm{Mg}^{2+}$, alone or in combination, were negative. This implies that the putative kinase phosphorylating WDA prefers $\mathrm{Mn}^{2+}$, as in the case of Pkn2 protein kinase of Myxococcus xanthus or Mbk of Mycobacterium tuberculosis (Udo et al., 1997; Peirs et al., 1997). We also observed a faint band in the test reaction carried out with the cytoplasmic fraction and WDA (Fig. 5d, lane 4). This could be due to contamination of the membrane fractions caused by disruption of the mycelia by sonication. According to these results, if the putative kinase phosphorylating WDA in T. curvata is membrane-bound then it would be strikingly similar to Pkg2, as the latter is also membranespanning (Nadvornik et al., 1999). It is tempting to speculate that PkwA could be a member of a signalling pathway occurring in early exponential stages of growth derived from spores of T. curvata. The phosphorylation of WDA and the presence of a putative protein Ser/Thr kinase domain in PkwA further strengthen this hypothesis. It is also very likely that the WD domain of PkwA could serve as a molecular switch in a phospho-relay signal transduction mechanism in T. curvata.

\section{ACKNOWLEDGEMENTS}

Preliminary sequence data for Chlorobium tepidum was obtained from The Institute for Genomic Research website at http://www.tigr.org (last accessed on 19 June 2000). This work was supported by grant 204/96/1262 from the Grant Agency of the Czech Republic. We are thankful to Dr Nadvornik for the gift of the pEX2 vector and anti-Pkg2 polyclonal antibody. We are also thankful to Dr Karel Drbal and Dr Ivan Hilgert for their valuable help in preparing $\mathrm{mAb}$ against the WD domain. We would like to thank Dr Miroslav Petricek for helpful discussion and Dr Radek Pelc for critical reading of the manuscript.

\section{REFERENCES}

Bhalerao, R. P., Salchert, K., Bako, L., Okresz, L., Szabados, L., Muranaka, T., Machida, Y., Schell, J. \& Koncz, C. (1999). Regulatory interaction of PRL1 WD protein with Arabidopsis SNF1-like protein kinases. Proc Natl Acad Sci USA 96, 5322-5327.

Bormann, E. R., Eikmanns, B. J. \& Sahm, H. (1992). Molecular analysis of the Corynebacterium glutamicum gdh gene encoding glutamate dehydrogenase. Mol Microbiol 6, 317-326.

Claney, C. E., Mendoza, M. G., Naismith, T. V., Komnan, M. F. \& Egelhoff, T. T. (1997). Identification of a protein kinase from Dictyostelium with homology to the novel catalytic domain of myosin heavy chain kinase A. J Biol Chem 272, 11812-11815.

Datta, P. K., Chytil, A., Gorska, A. E. \& Moses, H. L. (1998). Identification of STRAP, a novel WD domain protein in transforming growth factor-beta signaling. J Biol Chem 273, 34671-34674.

Fong, H. K., Hurley, J. B., Hopkins, R. S., Miake-Lye, R., Johnson, M. S., Doolittle, R. F. \& Simon, M. I. (1986). Repetitive segmental structure of the transducin beta subunit: homology with the CDC4 gene and identification of related mRNAs. Proc Natl Acad Sci U S A 83, 2162-2166.

Futey, L. M., Medley, Q. G., Cote, G. P. \& Egelhoff, T. T. (1995). Structural analysis of myosin heavy chain kinase A from Dictyostelium. J Biol Chem 270, 523-529.

Galfre, G., Howe, S. C., Milstein, C., Butcher, G. W. \& Howard, J. C. (1977). Antibodies to major histocompatibility antigens produced by hybrid cell lines. Nature 266, 550.

Harris, J. F., Hawley, R. G., Hawley, T. S. \& Crawford-Sharpe, G. C. (1992). Increased frequency of both total and specific monoclonal antibody producing hybridomas using a fusion partner that constitutively expresses recombinant IL-6. J Immunol Methods 148, 199-207.

Hoecker, U., Tepperman, J. M. \& Quail, P. H. (1999). SPA1, a WDrepeat protein specific to phytochrome A signal transduction. Science 284, 496-499.

Janda, L., Tichy, P., Spizek, J. \& Petricek, M. (1996). A deduced Thermomonospora curvata protein containing serine/threonine protein kinase and WD-repeat domains. J Bacteriol 178, 1487-1489.

Janda, L., Pavelka, T., Tichy, P., Spizek, J. \& Petricek, M. (1997). Production and properties of $\alpha$-glucosidase from the thermotolerant bacterium Thermomonospora curvata. J Appl Microbiol $83,470-476$. 
Johnson, L. N., Noble, E. M. \& Owen, D. J. (1996). Active and inactive protein kinases: structural basis for regulation. Cell $\mathbf{8 5}$, 149-158.

Kolman, M. F. \& Egelhoff, T. T. (1997). Dictyostelium myosin heavy chain kinase A subdomains. Coiled-coil and WD repeat roles in oligomerization and substrate targeting. J Biol Chem 272, 16904-16910.

Kwak, J. M., Kim, S. A., Lee, S. K., Oh, S. A., Byoun, C. H., Han, J. K. \& Nam, H. G. (1997). Insulin-induced maturation of Xenopus oocytes is inhibited by microinjection of a Brassica napus cDNA clone with high similarity to a mammalian receptor for activated protein kinase C. Planta 201, 245-251.

Laemmli, U. K. (1970). Cleavage of structural proteins during the assembly of the head of bacteriophage T4. Nature 227, 680-685.

Mannai, M. \& Cozzone, A. J. (1982). Endogenous protein phosphorylation in Escherichia coli extracts. Biochem Biophys Res Commun 107, 981-988.

Matsumoto, A., Hong, S.-K., Ishizuka, H., Horinouchi, S. \& Beppu, T. (1994). Phosphorylation of the AfsR protein involved in secondary metabolism in Streptomyces species by a eukaryotictype protein kinase. Gene 146, 47-56.

Nadvornik, R., Vomastek, T., Janecek, J., Technikova, Z. \& Branny, P. (1999). Pkg2, a novel transmembrane protein Ser/Thr kinase of Streptomyces granaticolor. J Bacteriol 181, 15-23.

Neer, E. J. \& Smith, T. F. (1996). G protein heterodimers: new structures propel new questions. Cell 84, 175-178.

Neer, E. J., Schmidt, C. J., Nambudripad, R. \& Smith, T. F. (1994). The ancient regulatory-protein family of WD-repeat proteins. Nature 371, 297-300.

Peirs, P., De Wit, L., Braibant, M., Huygen, K. \& Content, J. (1997). A serine/threonine protein kinase from Mycobacterium tuberculosis. Eur J Biochem 244, 604-612.

Petricek, M., Stajner, K. \& Tichy, P. (1989). Cloning of a thermostable $\alpha$-amylase gene from Thermomonospora curvata and its expression in Streptomyces lividans. J Gen Microbiol 135, 3303-3309.

Petricek, M., Tichy, P. \& Kuncova, M. (1992). Characterization of the $\alpha$-amylase-encoding gene from Thermomonospora curvata. Gene 112, 77-83.
Ron, D., Chen, C.-H., Caldwell, J., Jamieson, L., Orr, E. \& MochlyRosen, D. (1994). Cloning of an intracellular receptor for protein kinase C: a homolog of the $\beta$ subunit of G proteins. Proc Natl Acad Sci US A 91, 839-843.

Ron, D., Luo, J. \& Mochly-Rosen, D. (1995). C2 region-derived peptides inhibit translocation and function of $\beta$ protein kinase $\mathrm{C}$ in vivo. J Biol Chem 270, 24180-24187.

Sambrook, J., Fritsch, E. F. \& Maniatis, T. (1989). Molecular Cloning: a Laboratory Manual, 2nd edn. Cold Spring Harbor, NY: Cold Spring Harbor Laboratory.

Sheaff, R. J., Groudine, M., Gordon, M., Roberts, J. M. \& Clurman, B. E. (1997). Cyclin E-CKD2 is a regulator of $\mathrm{p} 27^{\mathrm{Kip} 1}$. Genes Dev 11, 1464-1478.

Smith, T. F., Gaitatzes, C., Suxena, K. \& Neer, E. J. (1999). The WD repeat: a common architecture for diverse functions. Trends Biochem Sci 24, 181-185.

Udo, H., Inouye, M. \& Inouye, S. (1997). Biochemical characterization of $\mathrm{Pkn} 2$, a protein serine/threonine kinase from Myxococcus xanthus, a Gram-negative developmental bacterium. FEBS Lett 400, 188-192.

Urabe, H. \& Ogawara, H. (1995). Cloning, sequencing and expression of serine/threonine kinase-encoding genes from Streptomyces coelicolor A3(2). Gene 153, 99-104.

Vomastek, T., Nadvornik, R., Janecek, J., Technikova, Z., Weiser, J. \& Branny, P. (1998). Characterisation of two putative protein Ser/Thr kinases from actinomycete Streptomyces granaticolor both endowed with different properties. Eur J Biochem 257, 55-61.

Yam, C. H., Ng, R. W. M., Siu, W. Y., Lau, A. W. S. \& Poon, R. Y. C. (1999). Regulation of cyclin A-Cdk2 by SCF component Skp1 and F-box protein Skp2. Mol Cell Biol 19, 635-645.

Zhang, C.-C. (1996). Bacterial signalling involving eukaryotic-type protein kinases. Mol Microbiol 20, 9-15.

Zhang, H., Hannon, G. H. \& Beach, D. (1994). p21-containing cycline kinases exist in both active and inactive states. Genes Dev 8, 1750-1758.

Received 28 June 2000; revised 4 August 2000; accepted 30 August 2000. 